date14 March 2021

\title{
A site-selective antiferromagnetic ground state in layered pnictide-oxide $\mathrm{BaTi}_{2} \mathrm{As}_{2} \mathrm{O}$
}

\author{
Xiang-Long Yu, ${ }^{1,2}$ Da-Yong Liu, ${ }^{1}$ Ya-Min Quan, ${ }^{1}$ Ting Jia, ${ }^{1}$ Hai-Qing Lin, ${ }^{3}$ and Liang-Jian Zou ${ }^{1, a)}$ \\ ${ }^{1)}$ Key Laboratory of Materials Physics, Institute of Solid State Physics, Chinese Academy of Sciences, \\ P. O. Box 1129, Hefei 230031, China \\ ${ }^{2)}$ University of Chinese Academy of Sciences, Beijing 100000, China \\ ${ }^{3)}$ Beijing Computational Science Research Center, Beijing 100084, China
}

The electronic and magnetic properties of $\mathrm{BaTi}_{2} \mathrm{As}_{2} \mathrm{O}$ have been investigated using both the first-principles and analytical methods. The full-potential linearized augmented plane-wave calculations show that the most stable state is a site-selective antiferromagnetic (AFM) metal with a $2 \times 1 \times 1$ magnetic unit cell containing two nonmagnetic Ti atoms and two other Ti atoms with antiparallel moments. Further analysis to Fermi surface and spin susceptibility shows that the site-selective AFM ground state is driven by the Fermi surface nesting and the Coulomb correlation. Meanwhile, the charge density distribution remains uniform, suggesting that the phase transition at $200 \mathrm{~K}$ in experiment is a spin-density-wave (SDW) transition.

PACS numbers: 31.15.A-, 75.10.Jm, 74.20Mn, 75.40.Cx

\section{INTRODUCTION AND NUMERICAL METHOD}

Recently Wang et al. synthesized a new layered pnictide oxide $\mathrm{BaTi}_{2} \mathrm{As}_{2} \mathrm{O}^{1}$. This compound is isostructural to $\mathrm{BaTi}_{2} \mathrm{Sb}_{2} \mathrm{O}$ and $\mathrm{Na}_{2} \mathrm{Ti}_{2} P n_{2} \mathrm{O}(P n=\mathrm{As}, \mathrm{Sb})$. These four pnictide oxides exhibit similar anomalies in magnetic susceptibility and electrical resistivity at a certain temperature $T_{p} \underline{2}-\underline{4}$. This shows that a phase transition occurs around $T_{p}$, very analogous to the prototype $\mathrm{LaFeAsO}^{5}$. The anomaly of $\mathrm{BaTi}_{2} \mathrm{As}_{2} \mathrm{O}$ at $200 \mathrm{~K}$ is ascribed to a possible spin-density-wave (SDW) or charge-densitywave (CDW) transition $\underline{1}$. However, the occurrence of a CDW state is usually accompanied by the distortion of crystal lattice ${ }^{7}$. The distortion was not clearly observed in the structure measurement. It is probable that the ground state is an SDW phase. Nevertheless, its groundstate electronic and magnetic structures remain an open question. In this paper we study the electronic and magnetic properties of $\mathrm{BaTi}_{2} \mathrm{As}_{2} \mathrm{O}$ by combining the first-principles electronic structure calculations and the analytical method. Our study on $\mathrm{BaTi}_{2} \mathrm{As}_{2} \mathrm{O}$ will not only uncover the underlying mechanism for its anomalous properties, but also address the properties of the isostructural compounds $\mathrm{BaTi}_{2} \mathrm{Sb}_{2} \mathrm{O}$ and $\mathrm{Na}_{2} \mathrm{Ti}_{2} \mathrm{Pn}_{2} \mathrm{O}$.

The electronic structure calculations are performed by using the full-potential linearized augmented planewave (FPLAPW) code of the package WIEN2K. The lattice parameters of $\mathrm{BaTi}_{2} \mathrm{As}_{2} \mathrm{O}(a=4.045608 \AA, c=$ $7.27228 \AA$ ) for our calculations are provided by Wang

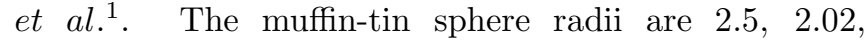
2.4 and $1.79 \mathrm{Bohr}$ for $\mathrm{Ba}$, $\mathrm{Ti}$, As and $\mathrm{O}$ atoms, respectively. The cut-off parameter $\mathrm{R}_{m t} \mathrm{~K}_{\max }$ is chosen to be 7.0. Our electronic structure calculations are

\footnotetext{
a) Author to whom correspondence should be addressed. Electronic mail: zou@theory.issp.ac.cn
}

based on the Perdew-Barke-Ernzerhof generalized gradient approximation (GGA) $\underline{\underline{6}}$ and its correlation correction $(\mathrm{GGA}+\mathrm{U})$. The number of $\mathbf{k}$ points is taken 2000 for unit cell and 1000 for supercell. Referring to the realistic magnetic structure of parent phase of iron-based superconductors ${ }^{8-11}$, we construct seven kinds of initial magnetic structures as possible candidates, including nonmagnetic (NM), ferromagnetic (FM), Néel antiferromagnetic (NAFM), striped AFM(SAFM), blocked checkerboard AFM (BCAFM), bi-collinear AFM (BAFM) and zigzag AFM (ZAFM) states. The last three configurations are shown in Fig. 1 (a)-(c), respectively.

\section{NUMERICAL RESULTS AND DISCUSSIONS}

Within the GGA scheme, we first find that FM, NAFM and SAFM states are unstable and they all converge to the NM state without spontaneous magnetization. The BCAFM order is stable. However, both the BAFM and ZAFM orders are unstable and the system converges to a particular AFM order, corresponding to such a $2 \times 1 \times 1$ magnetic unit cell which contains two titanium atoms without magnetic moments and two others with antiparallel moments, as shown in Fig. 1(d). In such a magnetic structure the AFM moments of Ti atoms are siteselective. More importantly, we find that the BCAFM and site-selective AFM phases are almost degenerate to the NM phase in total energy. The relative total energies per Ti atom referencing to the NM phase and the magnetic moment around each Ti atom are listed in Line (a) in Table I.

For these almost degenerate phases, we expect that electronic correlation would remove the degeneracy. Thus the GGA $+U$ calculations are performed for the different effective Coulomb correlation $U_{\text {eff }}=U-J$ where $U$ and $J$ are the on-site Coulomb and Hund's exchange 

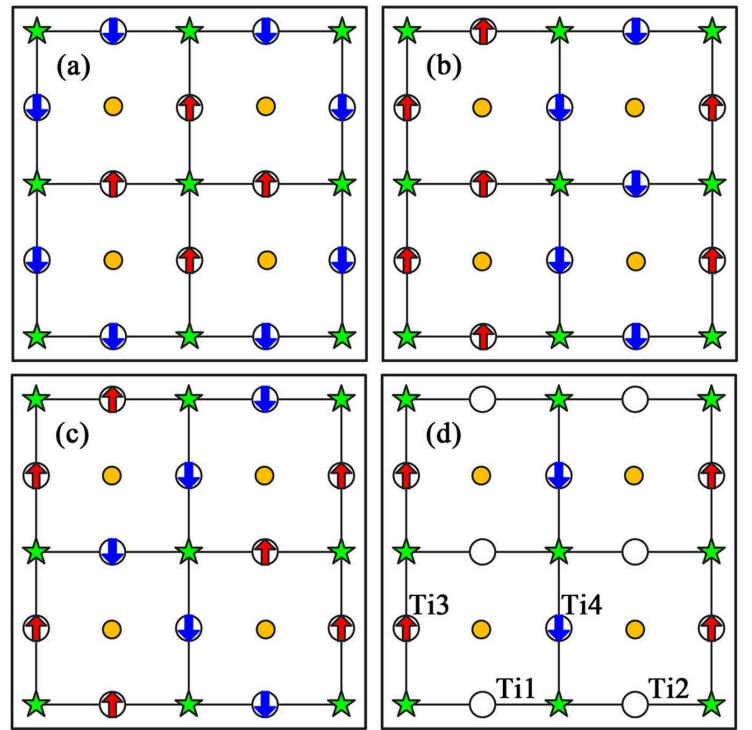

FIG. 1. (Color online) Four magnetic structures on $\mathrm{Ti}_{2} \mathrm{As}_{2} \mathrm{O}$ layer: (a) BCAFM, (b) BAFM, (c) ZAFM and (d) siteselective AFM ordered phases. The big and small circles represent $\mathrm{Ti}$ and $\mathrm{O}$ atoms, respectively; and the green star represents As atom.

interactions, respectively. Unlike the GGA results, the FM and NAFM phases are stable, but have relatively high total energies. The SAFM phase remains unstable. The BAFM and ZAFM phases are still unstable and both converge to the site-selective AFM state. On the other hand, the consideration of electronic correlation correction $U_{\text {eff }}$ lifts the degeneracy of the NM, BCAFM and site-selective AFM states, and the total energy of the siteselective AFM state becomes the lowest. Lines (b) and (c) in Table I summarize the relative total energies and magnetic moments for $U_{\text {eff }}=2$ and $3 \mathrm{eV}$. Meanwhile, one notices that several bands cross Fermi level for different $U_{\text {eff }}$ in the lowest energy state, suggesting that the site-selective AFM phase is metallic, in agreement with the experimental observation 1 .

To further explore the underlying mechanism of the phase transition at $T_{p}=200 \mathrm{~K}$, we have analyzed the electronic structures of the NM which corresponds to the high-symmetry phase at high temperature. The holetype and electron-type Fermi surfaces without electronic correlation correction are illustrated in Fig. 2 (a) and (b). The electron-type Fermi surface sheets around the corner of Brillouin zone ( $M$ point) can almost perfectly coincide with each other after the translation along the diagonal direction. It reveals a Fermi surface nesting with the nesting vector $\mathbf{Q}_{F 1} \approx\left(0.24 \frac{2 \pi}{a}, \pm 0.24 \frac{2 \pi}{a}, 0\right)$. Besides, there is an imperfect nesting between electrontype and hole-type Fermi surface sheets along $(1,0,0)$ or $(0,1,0)$, the corresponding nesting vector is $\mathbf{Q}_{F 2}=$ $\left(\frac{\pi}{a}, 0,0\right)$ or $\left(0, \frac{\pi}{a}, 0\right)$. Such two Fermi surface nestings would likely lead to an SDW instability in association with the observed anomalies in the experiment. Never-
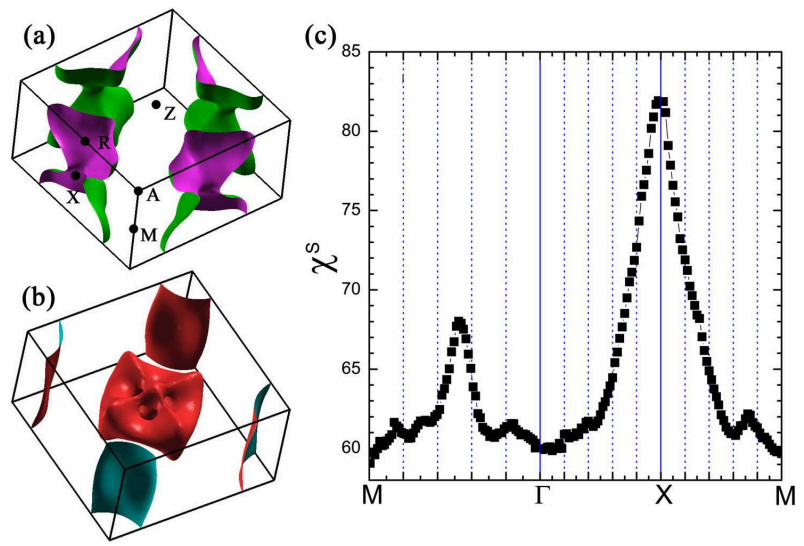

FIG. 2. (Color online) Fermi surfaces and spin susceptibility of $\mathrm{BaTi}_{2} \mathrm{As}_{2} \mathrm{O}$ in NM state within the GGA sheme: (a) hole-type Fermi surface sheets, (b) electron-type Fermi surface sheets and (c) the real part of $\chi^{S}$ with arbitrary unit.

theless, we need to perform further analysis to uniquely determine the realistic Fermi surface nesting vector that the SDW oscillates as $\mathbf{M} \cdot \cos \left(\mathbf{Q}_{F} \cdot \mathbf{R}\right)$ on the Ti-Ti square lattice in $\mathrm{BaTi}_{2} \mathrm{As}_{2} \mathrm{O}$.

In order to clarify which one of the above two nesting vectors dominates the ground-state magnetic structure, we have studied the spin susceptibility

$$
\chi^{S}(\mathbf{q})=-\frac{1}{N} \sum_{k, m, n} \frac{f\left(\varepsilon_{m}(\mathbf{k}+\mathbf{q})\right)-f\left(\varepsilon_{n}(\mathbf{k})\right)}{\varepsilon_{m}(\mathbf{k}+\mathbf{q})-\varepsilon_{n}(\mathbf{k})+i \eta} .
$$

The real part of $\chi^{S}$ along $M-\Gamma-X-M$ is shown in Fig. 2 (c). There are two peaks almost at halfway of the $\Gamma-M$ length and at the $X$ point, respectively. They are in well agreement with the above two nesting vectors. At the same time, we notice that the second peak, corresponding to the wavevector $\left(\frac{\pi}{a}, 0,0\right)$ or $\left(0, \frac{\pi}{a}, 0\right)$, is dominant. Therefore, it can be expected that $\mathbf{Q}_{F 2}$ may determine the ground-state magnetic structure.

Moreover, we have studied the electronic structures of the NM phase by using GGA $+U$ approach. When the effective Coulomb correlation $U_{\text {eff }}$ on $\mathrm{Ti}$ atom increases from 2 to $3 \mathrm{eV}$, there is no significant qualitative change, including the Fermi surface nesting and spin susceptibility. It is worthy of noticing that the site-selective AFM order, which corresponds to the lowest energy state in the GGA $+U$ calculations, just meets the Fermi surface nesting vector $\mathbf{Q}_{F 2}$ obtained in the NM state. This further confirms that the ground state of $\mathrm{BaTi}_{2} \mathrm{As}_{2} \mathrm{O}$ is the site-selective AFM phase. And considering the electronic correlation strength in other iron pnictides, we think that $U_{\text {eff }}=2 \mathrm{eV}$ is a proper Coulomb correlation. For such a correlation the energy of the site-selective ground state is lower about $12.5 \mathrm{meV} / \mathrm{Ti}$ than that of the $\mathrm{NM}$ state. Such an energy difference is in good agreement with the phase transition temperature $200 \mathrm{~K}$.

In Fig. 3 we have presented the electronic structures of the site-selective AFM ground state in the correlation 
TABLE I. Relative total energy per Ti atom and magnetic moment around each Ti atom in different magnetic states with $U_{\text {eff }}=0 \mathrm{eV}(\mathrm{a}), 2 \mathrm{eV}$ (b) and $3 \mathrm{eV}$ (c). The bar " - " means that the corresponding magnetic state is unstable and will converge to an NM state.

\begin{tabular}{|c|c|c|c|c|c|c|}
\hline & Magnetic structure & NM & FM & NAFM & BCAFM & site-selective AFM \\
\hline \multirow[t]{2}{*}{ (a) $U_{e f f}=0 \mathrm{eV}$} & Relative energy (meV) & 0 & - & - & -0.50 & -0.44 \\
\hline & Ti moment $\left(\mu_{B}\right)$ & 0 & - & - & 0.144 & 0.193 \\
\hline \multirow[t]{2}{*}{ (b) $U_{e f f}=2 \mathrm{eV}$} & Relative energy (meV) & 0 & -0.92 & -1.67 & -12.13 & -12.50 \\
\hline & Ti moment $\left(\mu_{B}\right)$ & 0 & 0.370 & 0.071 & 0.384 & 0.538 \\
\hline \multirow[t]{2}{*}{ (c) $U_{e f f}=3 \mathrm{eV}$} & Relative energy (meV) & 0 & $\overline{-13.74}$ & $\overline{-14.10}$ & -25.31 & -28.19 \\
\hline & Ti moment $\left(\mu_{B}\right)$ & 0 & 0.516 & 0.053 & 0.490 & 0.684 \\
\hline
\end{tabular}

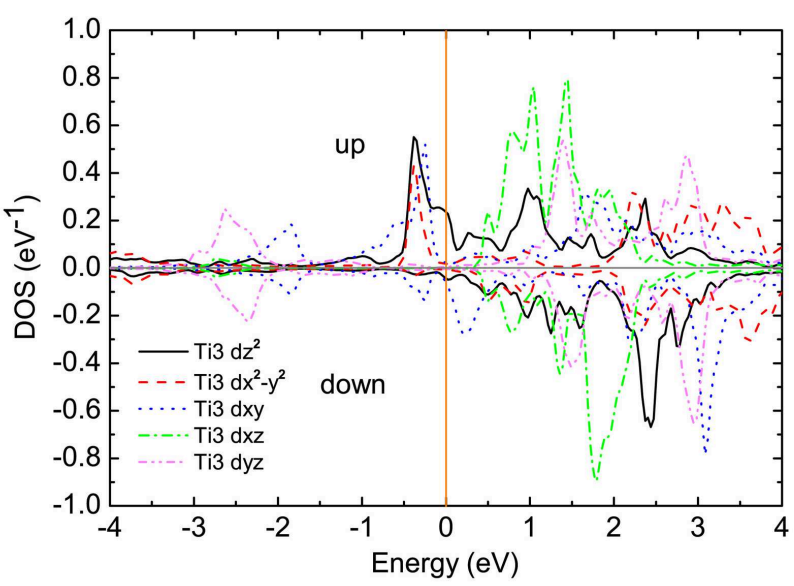

FIG. 3. (Color online) Density of states (DOS) projected into the Ti3 $-3 d$ orbitals for up and down spins within the $\mathrm{GGA}+\mathrm{U}$ scheme $\left(\mathrm{U}_{e f f}=2 \mathrm{eV}\right)$. Note that all DOS are depicted in the magnetic unit cell of site-selective AFM state with $U_{\text {eff }}=2 \mathrm{eV} . x, y$ and $z$ are directed along the $a, b$ and $c$ axis, respectively.

correction with $U_{\text {eff }}=2 \mathrm{eV}$. The greatest contribution to the density of states (DOS) at Fermi level is from $\mathrm{Ti}$ $3 d$ orbitals. It also confirms that Ti1 and Ti2 are NM, while Ti3 and Ti4 have antiparallel magnetic moments, about $0.54 \mu_{B}$. For Ti3 and Ti4 atoms, their partial DOS for up and down spins are almost equal except from -1.0 eV to $E_{F}$, as illustrated in Fig. 3. Such a splitting results in the formation of magnetic moments. Nearly localized $3 d_{x^{2}-y^{2}}$ and $3 d_{x y}$ electrons contribute to a part of magnetic moment, and itinerant $3 d_{z^{2}}$ electron also plays a key role in the formation of the magnetic order.

\section{CONCLUSION}

In summary, our detailed investigations on the electronic structure and spin susceptibility of $\mathrm{BaTi}_{2} \mathrm{As}_{2} \mathrm{O}$ have shown that both the Fermi surface nesting and Coulomb correlation drive the site-selective AFM metallic phase as the SDW-type ground state and the CDW order is excluded. We expect that the existence of the site-selective AFM order with a weak magnetic moment could be measured in future nuclear magnetic resonance experiment.

This work was supported by the NSF of China under Grant No.11104274 and 11204311, Knowledge Innovation Program of Chinese Academy of Sciences, and Director Grants of CASHIPS. Numerical calculations were performed in Center for Computational Science of CASHIPS.

${ }^{1}$ X. F. Wang, Y. J. Yan, J. J. Ying, Q. J. Li, M. Zhang, N. $\mathrm{Xu}$ and X. H. Chen, J. Phys.: Condens, Matter 22, 075702 (2010).

${ }^{2}$ P. Doan, M. Gooch, Z. Tang, B. Lorenz, A. Moeller, J. Tapp, P. C. W. Chu, and A. M. Guloy, J. Am. Chem. Soc. 134, 16520 (2012).

${ }^{3}$ T. Yajima, K. Nakano, F. Takeiri, T. Ono, Y. Hosokoshi, Y. Matsushita, J. Heister, Y. Kobayashi and H. Kageyama, J. Phys. Soc. Jpn. 81, 103706 (2012).

${ }^{4}$ A. Adam and H. U. Schuster, Z. Anorg. Allg. Chem. 584, 150 (1990).

${ }^{5}$ Y. Kamihara, T. Watanabe, M. Hirano, and H. Hosono, J. Am. Chem. Soc. 130, 3296 (2008).

${ }^{6}$ J. P. Perdew, K. Burke and M. Ernzerhof, Phys. Rev. Lett. 77, 3865 (1996).

${ }^{7}$ G. Grüner, Density Waves in Solids, pp. 31 (1994).

${ }^{8}$ X. W. Yan, M. Gao, Z. Y. Lu, and T. Xiang, Phys. Rev. B 83, 233205 (2011).

${ }^{9}$ W. Bao, Q. Z. Huang, G. F. Chen, M. A. Green, D. M. Wang, J. B. He, and Y. M. Qiu, Chin. Phys. Lett. 28, 086104 (2011).

${ }^{10}$ F. Ye, S. Chi, Wei Bao, X. F. Wang, J. J. Ying, X. H. Chen, H. D. Wang, C. H. Dong, and M. Fang, Phys. Rev. Lett. 107, 137003 (2011).

${ }^{11}$ W. Bao, Y. Qiu, Q. Huang, M. A. Green, P. Zajdel, M. R. Fitzsimmons, M. Zhernenkov, S. Chang, M. Fang, B. Qian, E. K. Vehstedt, J. Yang, H. M. Pham, L. Spinu, and Z. Q. Mao, Phys. Rev. Lett. 102, 247001 (2009). 